\title{
Phase dependence of Thomson scattering in an ultraintense laser field
}

\author{
Fei He, Y. Y. Lau, Donald P. Umstadter, and Trevor Strickler \\ Department of Nuclear Engineering and Radiological Sciences, University of Michigan, \\ Ann Arbor, Michigan 48109-2104
}

(Received 3 May 2002; accepted 23 July 2002)

\begin{abstract}
The Thomson scattering spectra of an electron by an ultraintense laser field are computed. It is found that the electron orbit, and therefore its nonlinear Thomson scattering spectra, depend critically on the amplitude of the ultraintense laser field and on the phase at which the electron sees the laser electric field. Contrary to some customary notions, the Thomson scattering spectra, in general, do not occur at integer multiples of the laser frequency and the maximum frequency is proportional to the first instead of the third power of the electric field strength for the case of an ultraintense laser. The implications of these findings are discussed. (C) 2002 American Institute of Physics.
\end{abstract}

[DOI: $10.1063 / 1.1507590]$

\section{INTRODUCTION}

The response of an electron under a steady state laser field is often the starting point in the construction of the dielectric function, in the formulation of the ponderomotive force, and in the calculation of various scattering or acceleration processes. As the laser intensity increases, various nonlinear phenomena emerge, ${ }^{1-4}$ such as "figure-8" orbits, ${ }^{5-9}$ nonlinear Thomson scattering, ${ }^{2,4-10}$ harmonic generation, ${ }^{2,6,10-16}$ self-focusing of the laser pulse in a plasma channel, ${ }^{17-20}$ etc. These nonlinear phenomena play key roles in proposed table-top accelerators and $\mathrm{x}$-ray sources. ${ }^{21,6,2}$ In the not too distant future, joules of laser energy will be focused down to a spot size of one wavelength $(\sim 1 \mu \mathrm{m})$, with the laser pulse length being compressed to the order of a few optical cycles. The electric field in such a laser pulse is extremely high; its normalized value, $a$ [defined just before Eq. (1)], is in the tens and beyond. The phase at which the electron experiences this pulse will become important because of the shortness of the laser pulse. In this paper, we examine the phase dependence of the electron orbits and of the resulting radiation spectra.

There are many instances in the interactions of intense lasers with matter where there can be a large spread in the initial phase angles $\left(\theta_{\text {in }}\right)$ of electrons with respect to the light wave. For instance, electrons that are born during the ionization of a gas will do so at different phases depending on the ionization potential. ${ }^{22}$ Finite plasma temperature will also lead to a spread in inital phase angles. Electrons will have different initial phases during the Compton scattering of intense light from a relativistic electron beam (at all collision angles except $180^{\circ}$ between the light beam and the electron beam). Electrons interacting with realistic three-dimensional focusing light pulses, with curved wave fronts, will also see different initial phases relative to other electrons that are at the same axial position but at a different radial position.

The electron orbit in an intense electromagnetic wave has been solved many times in the past. ${ }^{4-9}$ These works seldom address the dependence on the phase of the electromagnetic wave. A notable exception is the work of Gunn and
Ostriker. ${ }^{9}$ Here, we revisit this problem, concentrating on the varieties of the orbits and their resultant Thomson scattering spectra [Eq. (15)]. All calculations are in the lab frame. The effect of radiation damping is neglected.

\section{PHASE DEPENDENCE OF ELECTRON ORBITS}

Consider a linearly polarized wave, whose electric and magnetic fields are given by, respectively, $\mathbf{E}=\mathbf{x} E_{0} \cos \left(\omega_{0} t\right.$ $\left.-k_{0} z\right)$ and $\mathbf{B}=\mathbf{y} E_{0} \cos \left(\omega_{0} t-k_{0} z\right)$ in Gaussian units, where $\omega_{0}$ is the laser frequency, $k_{0}=\omega_{0} / c$, and $\mathbf{x}, \mathbf{y}$ are the unit vectors. Unless otherwise stated, we shall normalize time by $1 / \omega_{0}$, distance by $1 / k_{0}$, and velocity by $c$. In these units, the electric field strength is measured by the dimensionless parameter, $a=e E_{0} / m \omega_{0} c$. For electron motions in the $x-z$ plane, the relativistic Lorentz force law reads, in component form,

$$
\begin{aligned}
& d\left(\gamma \beta_{x}\right) / d t=a\left(1-\beta_{z}\right) \cos (t-z), \\
& d\left(\gamma \beta_{z}\right) / d t=a \beta_{x} \cos (t-z),
\end{aligned}
$$

where $\gamma=\left(1-\beta^{2}\right)^{-1 / 2}=\left(1-\beta_{x}^{2}-\beta_{z}^{2}\right)^{-1 / 2}$ is the relativistic mass factor with $\beta_{x}$ and $\beta_{z}$ being, respectively, the $x$ and $z$ components of the electron velocity (in units of $c$ ). Equations (1) and (2) lead to the energy conservation equation,

$d \gamma / d t=a \beta_{x} \cos (t-z)$.

Once the initial conditions for the electron trajectory are specified, the electron velocity $\boldsymbol{\beta}=\left(\beta_{x}, 0, \beta_{z}\right)$ and the electron displacement $\mathbf{r}=(x, 0, z)$ may be obtained as a function of $t$, according to Eqs. (1) and (2). However, it is more convenient to represent $\boldsymbol{\beta}, \mathbf{r}$, and $t$ parametrically in terms of $\theta$, defined by, ${ }^{5-9}$

$$
\theta=t-z \text {. }
$$

Upon subtracting Eqs. (2) and (3), we obtain the important relationship,

$$
\frac{1}{\gamma}=1-\beta_{z}=\frac{d \theta}{d t},
$$


where in writing the last expression, we have used Eq. (4). We have also assumed that at some instant of time, the electron is at rest. Call that instant of time $t=0$, at which the electron is located at $z=z_{\text {in }}$, whence $\theta=\theta_{\text {in }}=-z_{\text {in }}$ according to Eq. (4). Note from Eq. (5) that $\theta$ is a monotonically increasing function of $t$ and that there is a one-to-one correspondence between $t$ and $\theta$. Note also from Eq. (5) that $\beta_{z}$ $>0$ as $\gamma>1$. Thus, the electron always moves in the $+z$ direction (except when it is momentarily at rest).

In terms of $\theta$, one finds ${ }^{9}$

$$
\gamma=1+\frac{a^{2}}{2}\left(\sin \theta-\sin \theta_{\text {in }}\right)^{2},
$$

from which the $z$ velocity, $\beta_{z}=1-1 / \gamma$, and the $z$ momentum, $\gamma \beta_{z}=\gamma-1$, are then immediately known. They both depend on $\theta_{\text {in }}$. Likewise, one obtains the $x$ momentum, ${ }^{9}$

$$
\gamma \beta_{x}=a\left(\sin \theta-\sin \theta_{\text {in }}\right),
$$

and the $x$ coordinate,

$$
x=a\left[\left(\cos \theta_{\text {in }}-\cos \theta\right)-\left(\theta-\theta_{\text {in }}\right) \sin \theta_{\text {in }}\right] .
$$

The last term of Eq. (8) shows that, as $t$ increases (i.e., as $\theta$ increases), there is always a steady drift of the orbit in the $x$ direction except for very special values of $\theta_{\text {in }}$, that is, except for $\theta_{\text {in }}=n \pi, n=0$ or integer (Fig. 1). In the absence of this transverse drift, the motion is commonly called the "figure8 " motion, a term we shall still use here with this transverse drift. Note that in the case of large $a$ [e.g., $a=10$ in Figs. 1 (a) and $1(\mathrm{~b})]$ the shape of the figure- 8 orbit depends sharply on the phase, $\theta_{\text {in }}$. As we shall see in Eq. (16), these different shapes directly influence the fundamental frequency $\omega_{1}$ of the Thomson scattering spectrum through the net displacement, $\mathbf{r}_{0}$, that the electron undergoes in each figure- 8 orbit.

Finally, since $d t / d \theta=\gamma$ according to Eq. (5), we integrate Eq. (6) with respect to $\theta$ to obtain the parametric dependence of $t$,

$$
\begin{aligned}
t= & \left(\theta-\theta_{\text {in }}\right)\left[1+\frac{a^{2}}{2}\left(\frac{1}{2}+\sin ^{2} \theta_{\text {in }}\right)\right] \\
& +\frac{a^{2}}{2}\left[-\frac{\sin 2 \theta}{4}+2 \cos \theta \sin \theta_{\text {in }}-\frac{3 \sin 2 \theta_{\text {in }}}{4}\right] .
\end{aligned}
$$

The parametric dependence of the $z$ coordinate is given by $z=t-\theta$ in which $t$ is given by (9).

Thus, we have obtained the electron orbit, $\mathbf{r}$ and $\boldsymbol{\beta}$, parametrically in terms of $\theta$, which is related to the time $t$ by Eq. (9). These trajectories depend on $a$, and on the phase $\theta_{\text {in }}$. A sample of the trajectories is shown in Fig. 1. Equations (6) and (7) show that $\gamma$ and $\beta_{x}$ are periodic functions of $\theta$ with period $2 \pi$. So is $\beta_{z}$ by virtue of Eq. (5). The period, $T$, of this periodic ("figure- 8 ") motion is thus equal to the increase in $t$ as $\theta$ increases by $2 \pi$. From Eq. (9), one obtains ${ }^{9}$

$$
T=2 \pi\left[1+\frac{a^{2}}{2}\left(\frac{1}{2}+\sin ^{2} \theta_{\text {in }}\right)\right] .
$$

Over one orbital period, $T$, the electron undergoes a net displacement $\mathbf{r}_{0}=\left(x_{0}, 0, z_{0}\right)$ where $x_{0}$ is given by the increase in Eq. (8) as $\theta$ increases by $2 \pi$,
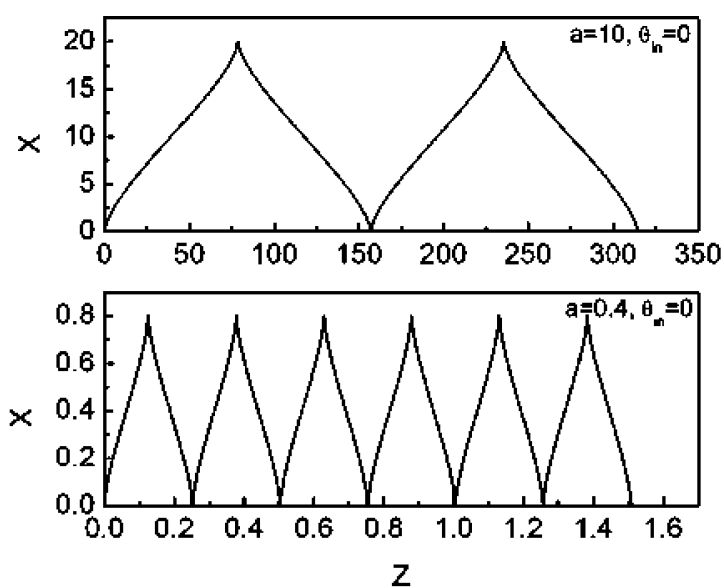

(a)

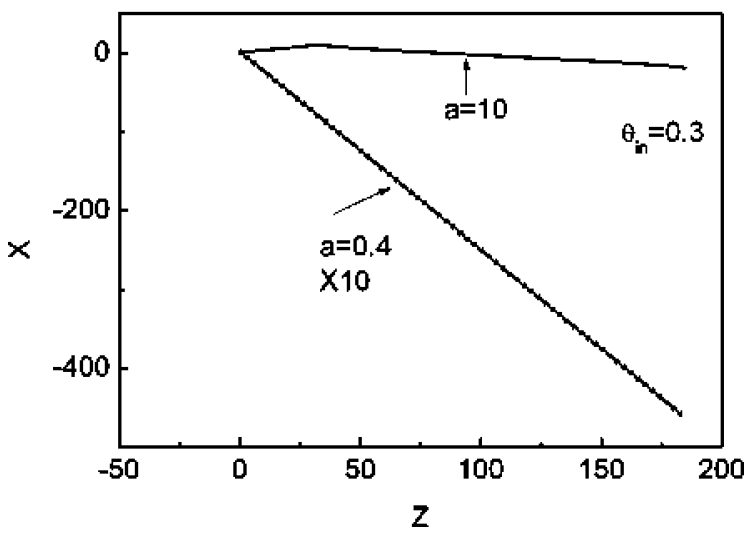

(b)

FIG. 1. Electron trajectories at various a and $\theta_{\text {in }}$. The laser wavelength is $2 \pi$ in the normalized scales. (a) $\theta_{\text {in }}=0$. Top: two figure- 8 orbits for $a$ $=10$. Bottom: six figure- 8 orbits for $a=0.4$. (b) $\theta_{\text {in }}=0.3$. Top curve shows one figure- 8 orbit for $a=10$. Bottom curve shows 63 figure- 8 orbits (magnified 10 times) for $a=0.4$

$$
x_{0}=-2 \pi a \sin \theta_{\text {in }},
$$

and $z_{0}$ is given by the increase in $z=t-\theta$ as $\theta$ increases by $2 \pi$,

$$
z_{0}=T-2 \pi=\pi a^{2}\left(\frac{1}{2}+\sin ^{2} \theta_{\text {in }}\right) .
$$

The dependence on $\theta_{\text {in }}$ of this net displacement, $\mathbf{r}_{0}$ $=\left(x_{0}, 0, z_{0}\right)$, and of the figure- 8 period, $T$, enters directly in the fundamental frequency $\omega_{1}$ of the Thomson scattering spectrum [Eqs. (15) and (16)].

\section{PHASE DEPENDENCE OF THOMSON SCATTERING SPECTRUM}

We next calculate the radiation spectrum that results from the electron acceleration. Once the electron displacement $\mathbf{r}$, and the normalized velocity $\boldsymbol{\beta}$, are known, its radiation spectrum is given by (in dimensional form) ${ }^{23}$

$$
\frac{d^{2} I}{d \Omega d \omega}=\frac{e^{2} \omega^{2}}{4 \pi^{2} c}|\mathbf{n} \times[\mathbf{n} \times \mathbf{F}(\omega)]|^{2},
$$




$$
\mathbf{F}(\omega)=\int_{-\infty}^{\infty} d t \boldsymbol{\beta}(t) e^{i \omega[t-\mathbf{n} \cdot \mathbf{r}(t) / c]}
$$

Equation (13) gives the energy radiated by the electron in the direction of the unit vector $\mathbf{n}$, per unit solid angle $\Omega$ per unit frequency $\omega$. Since $\boldsymbol{\beta}(t+T)=\boldsymbol{\beta}(t)$ and $\mathbf{r}(t+T)=\mathbf{r}(t)+\mathbf{r}_{0}$ (when written in dimensionless form), one may show from Eq. (14) that $\mathbf{F}(\omega)$ consists of an infinite series of delta function at the harmonics of $\omega_{1}$ :

$$
\mathbf{F}(\omega)=\sum_{m=-\infty}^{\infty} \mathbf{F}_{m} \delta\left(\omega-m \omega_{1}\right) .
$$

Here, $\omega_{1}$ is the fundamental frequency of the radiation spectrum. ${ }^{24}$ It is related to the laser frequency $\omega_{0}$ by

$$
\frac{\omega_{1}}{\omega_{0}}=\frac{2 \pi}{T-\mathbf{n} \cdot \mathbf{r}_{\mathbf{0}}},
$$

and $\mathbf{F}_{m}$ is a dimensionless vector given by

$$
\begin{aligned}
& \mathbf{F}_{m}=\frac{\omega_{1}}{\omega_{0}} \int_{\theta_{\text {in }}}^{\theta_{\text {in }}+2 \pi} d \theta \gamma \boldsymbol{\beta} e^{i m 2 \pi h(\theta),} \\
& h(\theta)=\frac{t(\theta)-\mathbf{n} \cdot \mathbf{r}(\theta)}{T-\mathbf{n} \cdot \mathbf{r}_{0}} .
\end{aligned}
$$

In Eqs. (16) and (17), $T$ is given by Eq. (10) and $\mathbf{r}_{0}$ $=\left(x_{0}, 0, z_{0}\right)$ is given by Eqs. (11) and (12). Note from Eqs. (10) and (16) that, for the case of an ultraintense laser field (large $a$ ), the fundamental frequency $\omega_{1}$ of the radiation depends mostly on $a, \theta_{\text {in }}$, and $\mathbf{n}$. The radiation spectrum extends to frequencies much lower than the laser frequency $\omega_{0}$. In fact, Eqs. (16) and (10) show that $\omega_{1} / \omega_{0} \sim 1 / a^{2}$ for $a^{2} \gg 1$. Figure 2 shows the dependence of the fundamental frequency $\omega_{1}$ in the backscattered direction $(\mathbf{n}=-\mathbf{z})$ as a function of $a$, for several values of $\theta_{\text {in }}$. Here, we see that for $a=10$, the fundamental frequency $\omega_{1}$ has a very large spread depending on $\theta_{\text {in }}$, but $\omega_{1}$ is only on the order of $1 \%$ of the laser frequency $\omega_{0}$. For small values of $a(a<0.3)$, Fig. 2 shows that $\omega_{1}$ approaches $\omega_{0}$, and the relative spread due to different $\theta_{\text {in }}$ is significantly less. Figure 3 is a threedimensional plot, showing the dependence of the fundamental frequency $\omega_{1}$ on the scattering direction $\mathbf{n}$.

Note that in the integral for $\mathbf{F}_{m}$ in Eq. (17), the integrand is a periodic function of $\theta$ with period $2 \pi$. Integrals of this type are efficiently computed using the Romberg method. ${ }^{25}$ Figures 4(a) and 4(b) show that the Thomson scattering spectrum is not at integer harmonics of the laser frequency. In fact, it depends on the phase $\theta_{\text {in }}$ even for the $a=0.4$ case as shown in Fig. 4(a). Note further that the Thomson scattering spectrum in Fig. 4(a) becomes exponentially small beyond the third harmonic of the laser frequency. For large values of $a[a=10$, Fig. 4(b)], the spectrum becomes almost continuous, the underlying reason is that the fundamental frequency, $\omega_{1}$, is only about $1 \%$ of the laser frequency, $\omega_{0}$ [Figs. 2 and 4(b)]. The dependence of the spectrum on the phase $\theta_{\text {in }}$ is apparent in Fig. 5, where we extend the spectrum of the $a$ $=10$ case in Fig. 4(b). Figure 5 shows that the Thomson

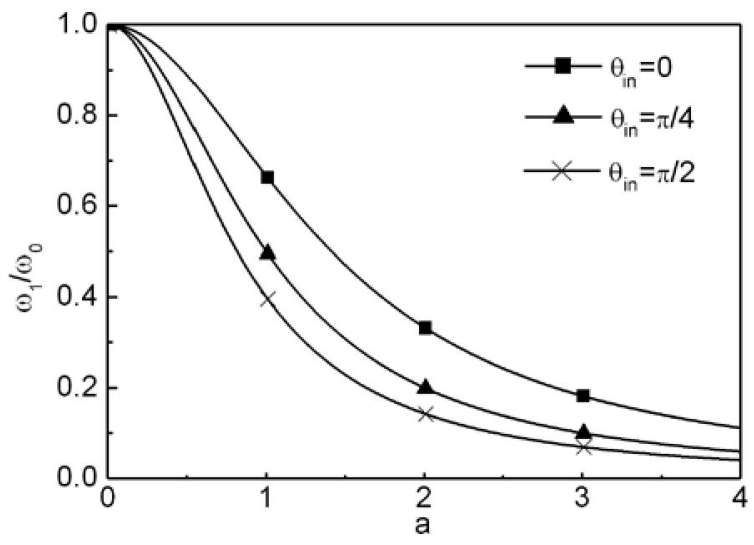

(a)

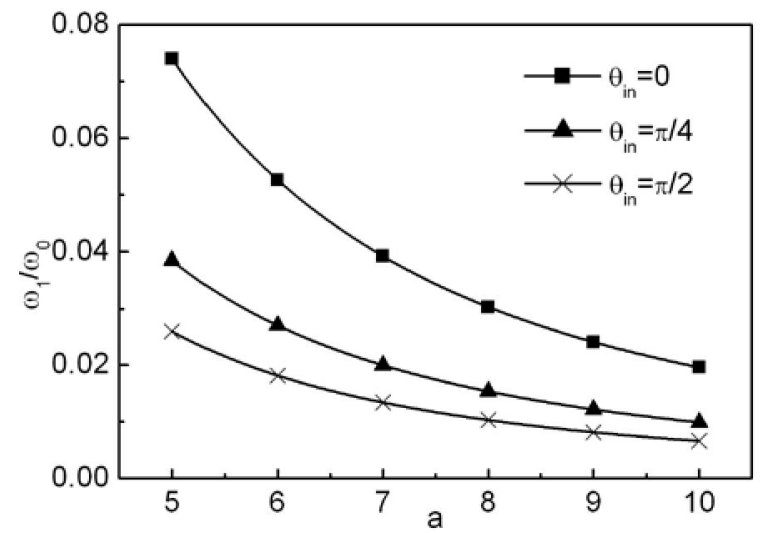

(b)

FIG. 2. Dependence of $\omega_{1} / \omega_{0}$, the normalized fundamental frequency of Thomson backscattered radiation, on the normalized laser field strength, $a$, for various $\theta_{\text {in }}$.

scattering spectrum peaks at $N \omega_{0}$, where $N$ is of order $a$ $=10$ (not $\left.a^{3}=1000\right)$. This is true for large $a$ regardless of the value of $\theta_{\text {in }}$.

The electron's orbital period $T$ is always larger than the laser's optical period. The latter equals $2 \pi$ in the dimensionless Eq. (10). Because of $T$ 's dependence on $\theta_{\text {in }}$, there is a relatively large spread of $T$ for large values of $a$ [Fig. 2]. This spread in $T$ leads to a spread in the fundamental frequency $\omega_{1}$ of the radiation spectrum [cf. Eq. (16)]. At high

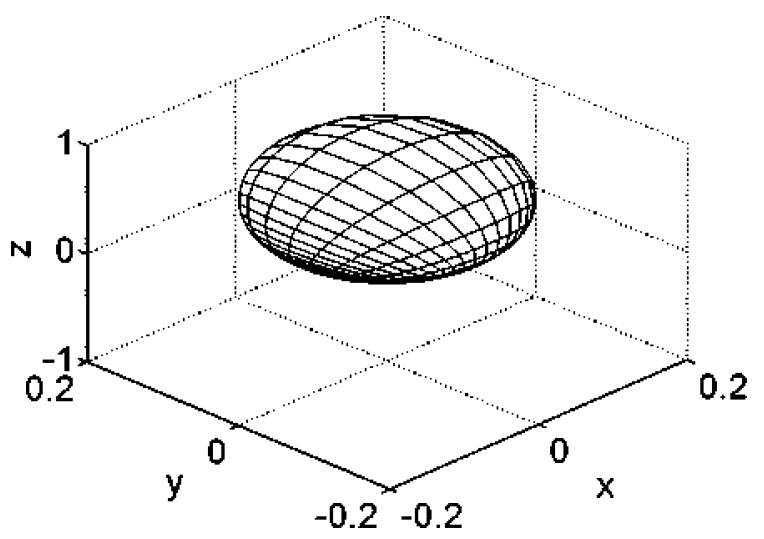

FIG. 3. Three-dimensional plot of $\omega_{1} / \omega_{0}$, whose value in any scattering direction is given by the numerical value of $\left(x^{2}+y^{2}+z^{2}\right)^{1 / 2}$ on the surface. 


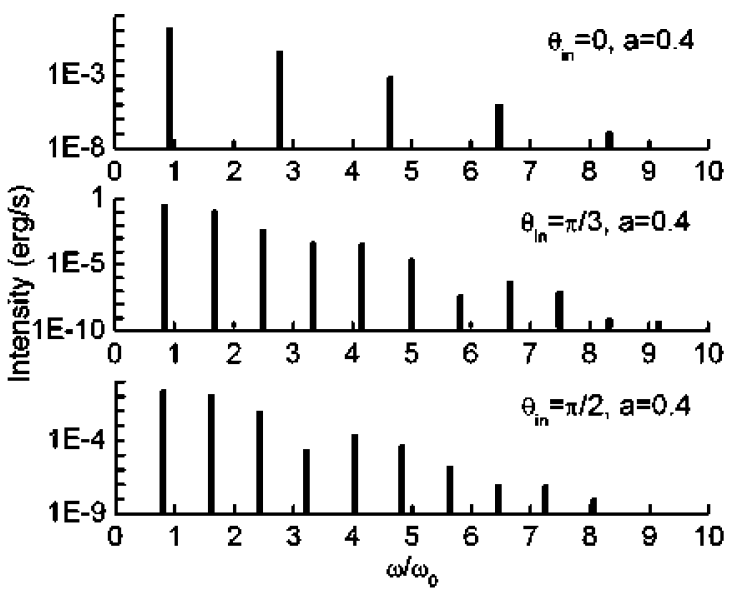

(a)

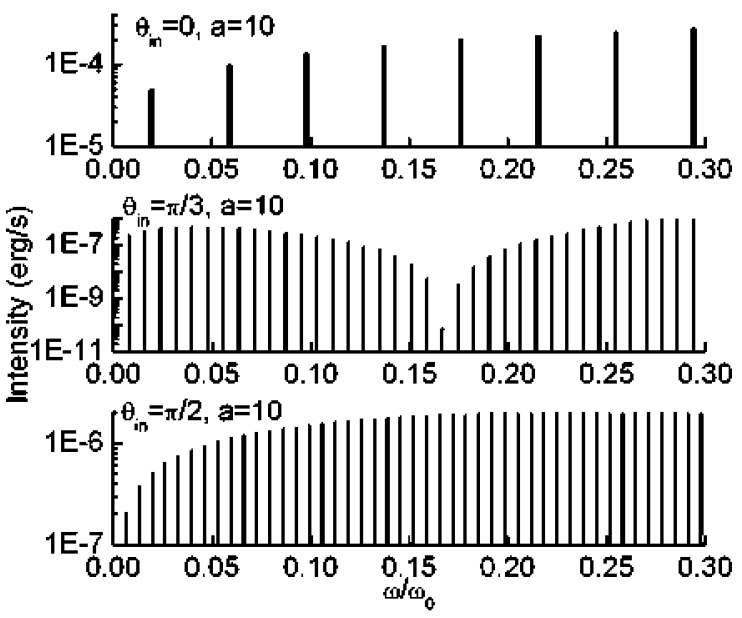

(b)

FIG. 4. Spectrum of Thomson backscattered radiation, $\left(e^{2} \omega^{2} / 4 \pi^{2} c\right) \mid \mathbf{n}$ $\times\left.\left(\mathbf{n} \times \mathbf{F}_{m}\right)\right|^{2}$, in erg/s, assuming $\lambda_{0}=2 \pi c / \omega_{0}=1 \mu \mathrm{m}$. (a) $a=0.4$. From top to bottom: $\theta_{\text {in }}=0, \pi / 3, \pi / 2$. (b) $a=10$. From top to bottom: $\theta_{\text {in }}$ $=0, \pi / 3, \pi / 2$.

harmonics of $\omega_{1}$, this spread is even more important, as easily seen from Eq. (15). This spread of $T$, however, is far less important if $a^{2} \ll 1$ [cf. Eq. (10)], and in this case, both the orbital frequency and the fundamental frequency of the radiation spectrum are close to the laser's optical frequency. Figure 4(a) also shows that only the first few harmonics of the laser frequency have appreciable scattered power for $a^{2}$ $\ll 1$.

According to conventional wisdom, ${ }^{6,15,2}$ the photonenergy $\left(E_{p}\right)$ scaling of light that is relativistically Thomson scattered should scale favorably with laser intensity, $E_{p}[\mathrm{eV}] \sim a^{3} / \lambda_{0}$, where $\lambda_{0}$ is the laser wavelength in microns. This implies that this mechanism might be used to efficiently generate $\mathrm{x}$ rays from visible light when $a$ is of order 10. However, this study indicates that the scaling is far less favorable in the deeply relativistic regime, $E_{p}[\mathrm{eV}]$ $\sim a / \lambda_{0}(\mu \mathrm{m})$, since the fundamental frequency, $\omega_{1} \sim 1 / a^{2}$. Compton scattering with a relativistic beam is thus more effective than relativistic Thomson scattering in generating $\mathrm{x}$ rays because the light effectively acts as an undulator in the former case but, as shown in this paper, not the latter case.

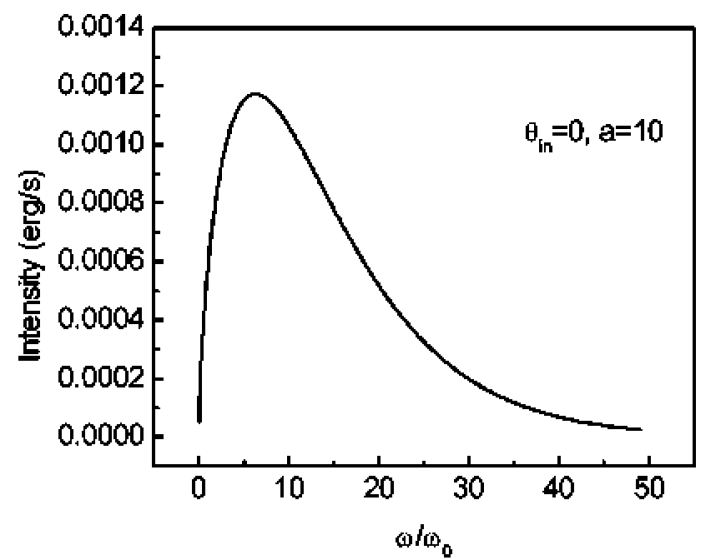

(a)

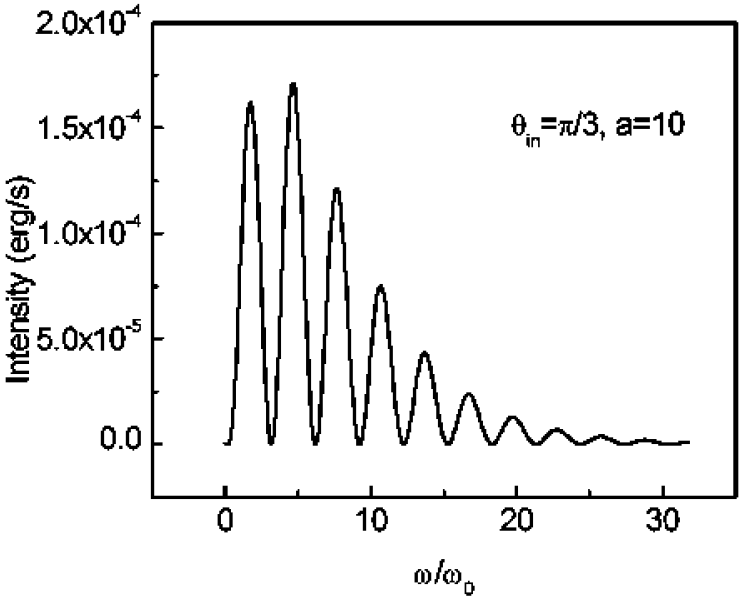

(b)

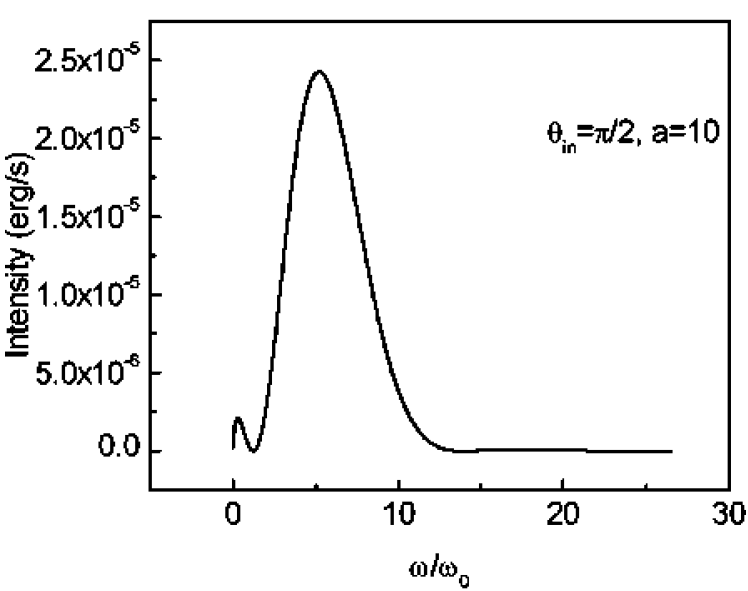

(c)

FIG. 5. The extended spectra for the $a=10$ cases in Fig. 4(b), showing peaks at $\omega=N \omega_{0}$, where $N=O(a)$ regardless of $\theta_{\text {in }}$. From top to bottom: $\theta_{\text {in }}=0, \pi / 3, \pi / 2$.

These qualitative results are anticipated from Gunn and Ostriker. $^{9}$

Since the optical wavelength equals $2 \pi$ in the dimensionless Eq. (11), the transverse displacement, $x_{0}$, in one figure-8 period is of order one optical wavelength for $a$ $\sim O(1)$. For large values of $a$, attainable with a very small laser spot size, an electron may be permanently displaced before it completes even one "figure-8" orbit. 
The above discussions naturally lead to an inquiry into the meaning of ponderomotive force in a tightly focused laser with $a^{2} \gg 1$. The ponderomotive force is typically derived under the assumptions that the electron's "wiggling motion" has the same period as the laser field, and an average is performed over an optical cycle. Here we see that, for the case of large $a$, the period $T$ of this "wiggling" motion much exceeds an optical period. Moreover, for large $a, \gamma$ $\gg 1$ during much of the figure- 8 orbit according to Eq. (6). The electron in a single figure- 8 orbit would stay mostly in phase with the laser electric field [and this is why the figure- 8 orbital period always exceeds the optical period, see Eq. (5)]. An average over the laser optical period then hardly makes sense. Similar inquiries could also be applied to plasma oscillation and the plasma dielectric function. For an ultraintense laser pulse that is highly localized in time and in space, the phase dependence and the large transverse excursion explored here need to be kept in mind.

Finally, we should point out that the calculation presented here has been extended to a circularly polarized wave. The spectra of Thomson scattering are similar to a linearly polarized wave. Higher dimensional effects are being evaluated.

\section{ACKNOWLEDGMENTS}

We thank Ronald Gilgenbach and Gerard Mourou for stimulating discussions.

This work was supported by the Office of Naval Research Grant N00014-01-1-0849, by the U.S. Department of Energy, Chemical Sciences, Geosciences and Biosciences Division of the Office of Basic Energy Sciences, Office of Science and by the U.S. National Science Foundation Grants Nos. 0114336 and 0078581.
${ }^{1}$ D. Umstadter and T. Norris, IEEE J. Quantum Electron. 33, 1878 (1997).

${ }^{2}$ D. Umstadter, Phys. Plasmas 8, 1774 (2001).

${ }^{3}$ R. W. Boyd, Nonlinear Optics (Academic, Boston, 1992).

${ }^{4}$ F. V. Hartemann, High Field Electrodynamics (CRC Press, Boca Raton, FL, 2001).

${ }^{5}$ E. S. Sarachik and G. T. Schappert, Phys. Rev. D 1, 2738 (1970).

${ }^{6}$ E. Esarey, S. K. Ride, and P. Sprangle, Phys. Rev. E 48, 3003 (1993).

${ }^{7}$ C. I. Castillo-Herrera and T. W. Johnston, IEEE Trans. Plasma Sci. 21, 125 (1993).

${ }^{8}$ F. V. Hartemann, Phys. Plasmas 5, 2037 (1998).

${ }^{9}$ J. E. Gunn and J. P. Ostriker, Astrophys. J. 165, 523 (1971).

${ }^{10}$ S. Y. Chen, A. Maksimchuk, and D. Umstadter, Nature (London) 396, 653 (1998).

${ }^{11}$ C. Bula, K. T. McDonald, E. J. Prebys et al., Phys. Rev. Lett. 76, 3116 (1996).

${ }^{12}$ S. Y. Chen, A. Maksimchuk, E. Esarey, and D. Umstadter, Phys. Rev. Lett. 84, 5528 (2000).

${ }^{13}$ G. Shvets, N. J. Fisch, and J.-M. Rax, Phys. Rev. Lett. 80, 2598 (1998).

${ }^{14}$ X. Liu, D. Umstadter, E. Esarey, and A. Ting, IEEE Trans. Plasma Sci. 21, 90 (1993)

${ }^{15}$ E. Esarey, A. Ting, P. Sprangle, D. Umstadter, and X. Liu, IEEE Trans. Plasma Sci. 21, 95 (1993).

${ }^{16}$ M. W. Walser, C. H. Keitel, A. Scrinzi, and T. Brabec, Phys. Rev. Lett. 85, 5082 (2000).

${ }^{17}$ P. Monot, T. Aguste, P. Gibbon et al., Phys. Rev. Lett. 74, 2953 (1995).

${ }^{18}$ S.- Y. Chen, G. S. Sarkisov, A. Maksimchuk, R. Wagner, and D. Umstadter, Phys. Rev. Lett. 80, 2610 (1998).

${ }^{19}$ A. B. Borisov, A. V. Borovskiy, V. V. Korobkin, A. M. Prokhorov, O. B. Shiryaev, X. M. Shi, T. S. Luk, A. McPherson, J. C. Solem, K. Boyer, and C. K. Rhodes, Phys. Rev. Lett. 68, 2309 (1992).

${ }^{20}$ R. Wagner, S. Y. Chen, A. Maksimchuk, and D. Umstadter, Phys. Rev. Lett. 78, 3125 (1997).

${ }^{21}$ J. S. Wurtele, Phys. Today 47, 33 (1994).

${ }^{22}$ P. B. Corkum, Phys. Rev. Lett. 71, 1994 (1993).

${ }^{23}$ J. D. Jackson, Classical Electrodynamics (Wiley, New York, 1962), p. 480.

${ }^{24}$ A. K. Puntajer and C. Leubner, Phys. Rev. A 40, 279 (1989).

${ }^{25}$ W. H. Press et al., Numerical Recipe in FORTRAN 77, 2nd ed. (Cambridge University Press, Cambridge, UK, 1996), Chap. 4. 